\title{
OPERATOR-VALUED FRAME GENERATORS FOR GROUP-LIKE UNITARY SYSTEMS
}

\author{
BIN MENG
}

\begin{abstract}
We investigate operator-valued frames with the structure of group-like unitary systems. We show that the commutant of a group-like unitary system can be characterized in terms of the analysis operators associated with all the operator-valued Bessel generators and give some sufficient and necessary conditions to describe when an operator-valued frame generator admits a Parseval dual. This extends work of J.Gabardo and D.Han but the proofs turn out to be more complicated. Then we characterize the Parseval operator-valued frame generators for certain unitary systems on a finite dimensional Hilbert space.
\end{abstract}

Mathematics subject classification (2010): 42C15, 46C05, 47B10.

Keywords and phrases: Operator-valued frame, group-like unitary system.

\section{REFERENCES}

[1] B. G. Bodmann, Optimal linear transmission by loss-insensitive packet encoding, Appl. Comput. Harmon. Anal. 22 (2007), 274-285.

[2] P. G. Casazza, O. Christensen, A. M. Lindner and R. Vershynin, Frames and the $\mathrm{Fe}$ ichtinger Conjecture, Proceedings AMS Vol. 133, No. 4 (2005), pp. 1025-1033.

[3] P. G. Cas azza And D. Edidin, Equivalents of the Kadison-Singer Problem, Contemp. Math. 435 (2007), 123-142.

[4] P. G. Casazza, J. Kovacevic, Equal-norm tight frames with erasures, Adv. Comp. Math. 18 (2003), 387-430.

[5] P. G. Cas azZa And G. Kutyniok, Frames of Subspaces, Contemporary Math 345 (2004), 87-114.

[6] P. G. CasazZA, G. Kutyniok, And S. Li, Fusion frames and distributed processing, Appl. Comput. Harmon. Anal. 25, 1 (2008), 114-132.

[7] I. DeAUBEChIES, Then lectures on wavelets, SIAM. Philadephia, 1992.

[8] M. Frank, D. LARSON, Frames in Hilbert $C^{*}$-modules and $C^{*}$-algebras, J. Operator Theory 48 (2002), 273-314.

[9] F. R. GANTMACHER, Matrix theory, vol. 1, Chelsea, 1959.

[10] J. Gabardo, D. HAn, Frame representations for group-like unitary operator systems, J. Operator Theory 49 (2003), 1-22.

[11] D. Han, D. LARSOn, Frames, Bases and group representations, Memoirs, AMS, 147 (2000), No. 694.

[12] D. HAn, P. LI, B. MENG AND W. TANG, Operator valued frames and structured quantum channels, to appear in Sci. in China.

[13] D. HAN, Frame representations and Parseval duals with applications to Gabor frames, Trans. Amer. Math. Soc. 360 (2008), 3307-3326.

[14] G. HaVE, Structure of the nth roots of a matrix, Lin. Alg. Appl. 187 (1993), 59-66.

[15] J. P. Gabardo, D. HAN, The uniqueness of the dual of Weyl-Heisenberg subspace frames, Appl. Comput. Harmon. Anal. 17 (2004), 226-240.

[16] R. B. Holmes, V. Paulsen, Optimal frame for erasures, Lin. Alg. Appl. 377 (2004), 31-51.

[17] V. Kaftal, D. Larson, S. Zhang, Operator-valued frames, Trans. Amer. Math. Soc. 361 (2009), 6349-6385.

[18] D. W. KRIBS, A quantum computing primer for operator theorists, Arxiv. math./0404553v2, 2004. 
[19] P. Lancaster, M. Tismenesky, The theory of matrices, Academic, 1985.

[20] E. LANCE, Hilbert $C^{*}$-modules, Cambridge Univ. Press, 1995.

[21] W. Sun, G-frames and g-Riesz bases, J. Math. Anal. Appl. 332, 1, 437-452. 